\title{
GENERALIZED LAGRANGE PROBLEMS IN THE CALCULUS OF VARIATIONS*
}

BY

C. F. ROOS $\dagger$

\section{INTRODUCTION}

In the new dynamical theory of economics there arises a very general problem which can be said to be a generalization of the Lagrange problem in the calculus of variations. $\neq$ It will not be necessary to consider the formulation of the corresponding economic theory here since I have already done this in another paper.§ It would hardly be fair, however, to introduce the reader to a rather unusual mathematical situation without giving some hint as to its origin. It seems desirable, therefore, to give first a brief economic formulation of the problem whose mathematical aspects will be discussed in this paper.

If there are two producers of an identical commodity $C$, manufacturing, respectively, amounts $u_{1}(x)$ and $u_{2}(x)$ of $C$ per unit time, subject to the respective cost functions $\phi_{1}\left(u_{1}, u_{1}^{\prime}, u_{2}, u_{2}^{\prime}, u_{3}, u_{3}^{\prime}, x\right)$ and $\phi_{2}\left(u_{1}, u_{1}^{\prime}, u_{2}, u_{2}^{\prime}\right.$, $\left.u_{3}, u_{3}^{\prime}, x\right)$, where $u_{3}(x)$ is the selling price of $C$ at a time $x$, then the respective profits obtained during an interval of time $x_{0} \leqq x \leqq x_{1}$ are

$$
\begin{aligned}
& I_{1}=\int_{x_{0}}^{x_{1}}\left[u_{3} u_{1}-\phi_{1}\left(u_{1}, u_{1}^{\prime}, u_{2}, u_{2}^{\prime}, u_{3}, u_{3}^{\prime}, x\right)\right] d x, \\
& I_{2}=\int_{x_{0}}^{x_{1}}\left[u_{3} u_{2}-\phi_{2}\left(u_{1}, u_{1}^{\prime}, u_{2}, u_{2}^{\prime}, u_{3}, u_{3}^{\prime}, x\right)\right] d x,
\end{aligned}
$$

where $\phi_{1}$ and $\phi_{2}$ are assumed to be continuous with their first and second derivatives with respect to all their arguments, and primes denote derivatives with respect to time $x$.

The rates of production $u_{1}(x)$ and $u_{2}(x)$ and the price $u_{3}(x)$ will satisfy an equation of demand which in the general case will be of the form

- Presented to the Society, December 31, 1926; received by the editors December 2, 1926.

$\dagger$ National Research Fellow in Mathematics.

$\ddagger$ For a special example of this problem, see C. F. Roos, A mathematical theory of competition, American Journal of Mathematics, vol. 47 (1925), pp. 163-175. See also G. C. Evans, The dymamics of monopoly, American Mathematical Monthly, vol. 31 (1921).

\& C. F. Roos, A dynamical theory of economics, Journal of Political Economy, vol. 35 (1927). See also Roos, Dynamical economics, Proceedings of the National Academy of Sciences, vol. 13 (1927). 


$$
G\left(u_{1}, u_{1}^{\prime}, \cdots, u_{3}^{\prime}, x\right)=\int_{x_{0}}^{x} P\left(u_{1}, u_{1}^{\prime}, \cdots, u_{3}^{\prime}, x, s\right) d s
$$

where $G$ and $P$ have continuity properties similar to those of $\phi_{1}$ and $\phi_{2}{ }^{*}$ Each manufacturer will consider his rate of production to be influenced by the rate of production of his competitor only through the equation of demand, and will desire to determine his own rate of production in such a way that he obtains a maximum profit over some interval of time, say $x_{0} \leqq x \leqq x_{1}$.

The problem of competition for this state of affairs will then be the problem of determining a curve $\Gamma$ in the space $\left(u_{1}, u_{2}, u_{3}, x\right)$, satisfying a functional equation (1), such that an integral $I_{1}$, taken along $\Gamma$ from $x_{0}$ to $x_{1}$, is a maximum when $u_{2}$ is momentarily held fixed, and such that a second integral $I_{2}$, also taken along $\Gamma$ from $x_{0}$ to $x_{1}$, is a maximum when $u_{1}$ is momentarily held fixed. In the usual case the initial time $x_{0}$ and the corresponding initial values of the $u_{i}, i=1,2,3$, are fixed. The end time $x_{1}$ and the corresponding end values of the $u_{i}$ may be regarded as fixed or not, depending upon the nature of the problem under consideration. Both cases will be considered at some length in the following paragraphs.

For the particular case $P \equiv 0$ the equation of demand becomes simply a first-order differential equation. For this case the problem of competition can be solved by the methods employed in the classical Lagrange problem in the calculus of variations. $f$ In order to obtain a solution in the classical way we need, however, two sets of Lagrange multipliers, and this makes the problem quite difficult. In the following pages I shall give an analysis for the case in which the rates of production and price are related by a differential equation of demand $G\left(u_{1}, u_{1}^{\prime}, u_{2}, u_{2}^{\prime}, u_{3}, u_{3}^{\prime}, x\right)=0$ without using multipliers, and shall obtain necessary and sufficient conditions. These conditions, although functional in character, seem simpler than the corresponding conditions which would be obtained by the classical analysis.

In discussing the Lagrange problem for several differential equations $G_{k}\left(u_{1}, u_{1}^{\prime}, \cdots, u_{n}, u_{n}^{\prime}, x\right)=0, k=1, \cdots, m<n$, I introduce the theory of Volterra integral equations into my analysis to replace the classical theory by means of multipliers. This use of the theory of integral equations enables me to obtain a method for solving the more general problem for which $P_{k}\left(u_{1}, u_{1}^{\prime}, \cdots, u_{n}, u_{n}^{\prime}, x, s\right) \not \equiv$. So far as I know this use of integral equa-

* Roos, Dynamical economics, loc. cit.

$\dagger$ J. Hadamard, Leģons sur le Calcul des Variations, pp. 217 and sequence. See also G. A. Bliss, The Problem of Lagrange in the Calculus of Variations, lectures given at the University of Chicago, summer quarter 1925, mimeographed by O. E. Brown, Northwestern University, Evanston, Illinois. 
tions is entirely new. As a result the following exposition, although lengthy, does not represent a complete treatment of the subject.

\section{FIXED END POINTS. EULERIAN EQUATIONS IN FUNCTIONAL FORM}

1. Geometrical interpretation of the problem. In order to make our analysis easier to follow let us first examine the problem for which both end points are fixed, and for which $P\left(u_{1}, u_{1}^{\prime}, \cdots, x, s\right) \equiv 0$, from a geometrical view point. In the hyperspace $\left(u_{1}, u_{2}, u_{3}, x\right)$ let $u_{2}=u_{2}(x)$ be any function, continuous with its first derivative, and substitute this value of $u_{2}$ in the integrand $F_{1}\left(u_{1}, u_{1}^{\prime}, \cdots, u_{3}^{\prime}, x\right)$ of an integral $I_{1}$, corresponding to the $I_{1}$ of the introduction, and in the differential equation $G=0$. The function $F_{1}$ becomes a function $F_{1}\left(u_{1}, u_{1}^{\prime}, u_{2}(x), u_{2}^{\prime}(x), u_{3}, u_{3}^{\prime}, x\right)$, and $G=0$ becomes a differential equation $G\left(u_{1}, u_{1}^{\prime}, u_{2}(x), u_{2}^{\prime}(x), u_{3}, u_{3}^{\prime}, x\right)=0$. The problem of finding $u_{1}=y_{1}(x)$ which maximizes $I_{1}$ is thus reduced to the problem of finding a function $y_{1}(x)$ which maximizes

$$
I_{1}=\int_{x_{0}}^{x_{1}} F_{1}\left(u_{1}, u_{1}^{\prime}, u_{2}(x), u_{2}^{\prime}(x), u_{3}, u_{3}^{\prime}, x\right) d x
$$

and satisfies $G=0$ and given end conditions whatever they may be.

Again, if $u_{1}(x)=y_{1}(x)$ be substituted in the integrand $F_{2}$ and in $G=0$, these become, respectively, $F_{1}\left(y_{1}(x), y_{1}^{\prime}(x), u_{2}, u_{2}^{\prime}, u_{3}, u_{3}^{\prime}, x\right)$ and $G\left(y_{1}(x)\right.$, $\left.y_{1}^{\prime}(x), u_{2}, u_{2}^{\prime}, u_{3}, u_{3}^{\prime}, x\right)=0$. Choosing the function $u_{2}(x)=y_{2}(x)$ so that it satisfies $G=0$ and maximizes

$$
I_{2}=\int_{x_{1}}^{x_{1}} F_{2}\left(y_{1}(x), y_{1}^{\prime}(x), u_{2}, u_{2}^{\prime}, u_{3}, u_{3}^{\prime}, x\right) d x
$$

completes the solution of the problem, for $u_{1}$ and $u_{3}$ have already been determined in terms of $u_{2}^{\prime}(x)$. It is important to note that we have assumed the existence of a solution without showing that one actually exists. Conditions for the existence of a solution will be discussed in Part IV of this paper.

2. Admissible arcs and variations. An arc $u_{i}=u_{i}(x), i=1,2,3$, which is continuous on the interval $x_{0} \leqq x \leqq x_{1}$, and is such that the interval can be divided into a finite number of subintervals on each of which the functions $u_{i}(x)$ have continuous derivatives up to and including those of the second order will be called an admissible arc. This definition will permit a maximizing arc to have a finite number of corners. All of the elements of an admissible arc shall be required to lie in a simply connected region of a hyperspace $\left(u_{1}, u_{2}, u_{3}, x\right)$, and to satisfy the differential equation $G\left(u_{1}, u_{1}^{\prime}, u_{2}, u_{2}^{\prime}, u_{3}\right.$. 
$\left.u_{3}^{\prime}, x\right)=0$, and, furthermore, to satisfy certain end conditions. ${ }^{*}$ In the following paragraphs all admissible arcs will be regarded as fixed at a fixed $x_{0}$, i.e.

$$
u_{1}\left(x_{0}\right)=u_{10}, u_{2}\left(x_{0}\right)=u_{20}, u_{8}\left(x_{0}\right)=u_{30},
$$

and either variable or fixed at $x_{1}$ depending upon the particular problem under consideration. The behavior of the arcs at $x_{1}$ will be pointed out as the work progresses.

If a two-parameter family of admissible arcs $u_{i}=u_{i}(x, a, b)$ containing a particular admissible arc $\Gamma$ for the parametric values $a=b=0$ be given, we shall call the functions

$$
\xi_{1}(x)=\partial u_{1}(x, 0,0) / \partial a, \xi_{2}(x)=\partial u_{2}(x, 0,0) / \partial b
$$

partial variations of the family along $\Gamma$. Ordinarily we would require a threeparameter family to cover the space $\left(u_{1}, u_{2}, u_{3}, x\right)$ completely, but the differential equation $G=0$ and the initial condition $u_{3}\left(x_{0}\right)=u_{30}$ removes one degree of freedom.

3. The Eulerian equations in functional form. Let us write

$$
\begin{aligned}
& u_{\alpha}=y_{\alpha}+\psi_{\alpha}(x, a, b) \\
& u_{3}=y_{3}+\theta(x, a, b),
\end{aligned}
$$$$
(\alpha=1,2),
$$

where the $\psi_{\alpha}$ are functions, continuous in $x, a$ and $b$, possessing continuous derivatives of the first order with respect to $x, a$ and $b$ and vanishing when $a$ and $b$ vanish. The functions $y_{\alpha}$ and $y_{3}$ are the functions $u_{i}(x), i=1,2,3$, defining the maximizing curve $\Gamma$ which we suppose for the present to exist a priori.

In our analysis we shall have to require that the functions $F_{1}, F_{2}$ and $G$ possess continuous derivatives of the second order with respect to each of the arguments $u_{i}, u_{i}^{\prime}, x, i=1,2,3$, and, furthermore, that $\partial G / \partial u_{3}^{\prime} \neq 0$ in the interval $x_{0} \leqq x \leqq x_{1}$. Under these hypotheses the function $\theta$ is determined by $G=0$ and the first two equations of (3), except for an arbitrary constant, as a continuous function of $x, a, b$ with continuous derivatives of the first order.

The derivatives $\partial \theta / \partial a$ and $\partial \theta / \partial b$ satisfy the equations of partial variations $\left(\partial G / \partial u_{1}\right) \partial \psi_{1} / \partial a+\left(\partial G / \partial u_{1}^{\prime}\right) \partial \psi_{1}^{\prime} / \partial a+\left(\partial G / \partial u_{3}\right) \partial \theta / \partial a+\left(\partial G / \partial u_{3}^{\prime}\right) \partial \theta^{\prime} / \partial a=0$, $\left(\partial G / \partial u_{2}\right) \partial \psi_{2} / \partial b+\left(\partial G / \partial u_{2}^{\prime}\right) \partial \psi_{2}^{\prime} / \partial b+\left(\partial G / \partial u_{3}\right) \partial \theta / \partial b+\left(\partial G / \partial u_{3}^{\prime}\right) \partial \theta^{\prime} / \partial b=0$, and will, therefore, also be continuous and have continuous partial derivatives

* See Bliss, loc. cit., p. 3. 
of the first order, on account of the continuity requirements on $G$. We further restrict the $\psi_{\alpha}$ by the following conditions:

$$
\begin{array}{ll}
\partial \psi_{1} / \partial a=\xi_{1}(x), & \partial \psi_{1} / \partial b=0, \\
\partial \psi_{2} / \partial a=0, & \partial \psi_{2} / \partial b=\xi_{2}(x),
\end{array}
$$

when $a=b=0$. We employ the following notation: $\partial \theta / \partial a=\theta_{a}(x)$ and $\partial \theta / \partial b=\theta_{b}(x)$ when $a=b=0$.

Since we have assumed the end values of $u_{1}$ and $u_{2}$ to be fixed at $x_{1}$ as well as at $x_{0}$, we can write

$$
\xi_{1}\left(x_{0}\right)=\xi_{1}\left(x_{1}\right)=\xi_{2}\left(x_{0}\right)=\xi_{2}\left(x_{1}\right)=0 .
$$

For the parametric values $a=b=0$ the function $\theta(x, 0,0)=\theta(x)$ must satisfy the differential equations of partial variations

$$
\begin{aligned}
& \left(\partial G / \partial u_{1}\right) \xi_{1}+\left(\partial G / \partial u_{1}^{\prime}\right) \xi_{1}^{\prime}+\left(\partial G / \partial u_{3}\right) \theta_{a}+\left(\partial G / \partial u_{3}^{\prime}\right) \theta_{a}^{\prime}=0 \\
& \left(\partial G / \partial u_{2}\right) \xi_{2}+\left(\partial G / \partial u_{2}^{\prime}\right) \xi_{2}^{\prime}+\left(\partial G / \partial u_{3}\right) \theta_{b}+\left(\partial G / \partial u_{3}^{\prime}\right) \theta_{b}^{\prime}=0 .
\end{aligned}
$$

The first of these determines $\theta_{a}$ in terms of $\xi_{i}$ and the partial derivatives of $G$ with respect to $u_{1}$ and $u_{1}^{\prime}$, except for a constant, whereas the second determines $\theta_{b}$ in terms of $\xi_{2}$ and the partial derivatives of $G$ with respect to $u_{2}$ and $u_{2}^{\prime}$, except for a constant. Choosing these constants so that each of the partial variations $\theta_{a}\left(x_{0}\right)$ and $\theta_{b}\left(x_{0}\right)$ vanishes implies that the total variation of the function $\theta$ be zero at $x_{0}$, i.e. $\delta \theta=\theta_{a} \delta a+\theta_{b} \delta b=0$ at $x=x_{0}$. Conversely, since the $\psi_{\alpha}$ are arbitrary, the vanishing of $\delta \theta$ implies the vanishing of both $\theta_{a}$ and $\theta_{b}$. The equations (4) and the initial conditions (2), therefore, completely determine the variations of $u_{3}$. The functions $u_{1}$ and $u_{2}$ have thus been classified as independent functions in a manner similar to the way in which variables are classified in the ordinary theory of maxima and minima of functions.

If functions $u_{i}(x, a, b)$, defining a two-parameter family of admissible arcs containing $\Gamma$ for the parametric values $a=b=0$, are substituted in $I_{2}$. this integral becomes a function of $a$ and $b$ defined by

$$
I_{1}(a, b)=\int_{x_{n}}^{x_{1}} F_{1}\left(u_{1}(x, a, b), u_{1}^{\prime}(x, a, b), \cdots, u_{3}^{\prime}(x, a, b), x\right) d x .
$$

The partial variation of this integral with respect to $a$ reduces to

$$
\begin{gathered}
\left(\partial I_{1} / \partial a\right) \delta a=\int_{x_{0}}^{x_{1}}\left[\left(\partial F_{1} / \partial y_{1}\right) \xi_{1}\right. \\
+\left(\partial F_{1} / \partial y_{1}^{\prime}\right) \xi_{1}^{\prime}+\left(\partial F_{1} / \partial y_{3}\right) \theta_{a} \\
\left.+\left(\partial F_{1} / \partial y_{3}^{\prime}\right) \theta_{a}^{\prime}\right] d x \delta a
\end{gathered}
$$

for $a=b=0$. 
Instead of proceeding in the classical way we shall solve the differential equation (4) for $\theta_{a}$ and develop a theory without the use of Lagrange multipliers.* This procedure seems to be more directly an extension of the ordinary theory of maxima and minima; it allows us to obtain the Weierstrass, Legendre and Jacobi conditions by an analysis which is simpler than that used in the classical theory, and, furthermore, it leads to a method for solving the Lagrange problem when the differential equations are replaced by functional equations of the type (1). We proceed as follows:

Since by hypothesis $\partial G / \partial y_{3}^{\prime}$ is not zero in the interval $x_{0} \leqq x \leqq x_{1}$, the solution of (4) for $\theta_{a}$ is

$$
\theta_{a}=\int_{x_{0}}^{x} e^{V_{3}}\left[\left(\partial G_{3}^{\prime} / \partial y_{1}\right) \xi_{1}+\left(\partial G_{3}^{\prime} / \partial y_{1}^{\prime}\right) \xi_{1}^{\prime}\right] d t,
$$

where the following notation has been introduced: $\left(\partial G / \partial y_{3}\right) /\left(\partial G / \partial y_{s}^{\prime}\right)$ $=-\partial G_{3}^{\prime} / \partial y_{3} ; \quad\left(\partial G / \partial y_{1}\right) /\left(\partial G / \partial y_{3}^{\prime}\right)=-\partial G_{3}^{\prime} / \partial y_{1} ; \quad\left(\partial G / \partial y_{1}^{\prime}\right) /\left(\partial G / \partial y_{3}^{\prime}\right)=-$ $\partial G_{z}^{\prime} / \partial y_{1}^{\prime} ; V_{i}=\int_{t}^{x}\left(\partial G_{i}^{\prime} / \partial y_{i}\right) d s$. The assumption $\theta_{a}\left(x_{0}\right)=0$, made above, does not necessarily impose a limitation on this method, for, if $u_{3}$ were variable at $x_{0}$, the solution for $\theta_{a}$ would be the solution above plus the variation of $u_{3}$ at $x_{0}$.

Differentiation of (5) with respect to $x$ determines $\theta_{a}^{\prime}$ by the formula

$$
\begin{aligned}
\theta_{a}^{\prime}=\left(\partial G_{3}^{\prime} / \partial y_{1}\right) \xi_{1} & +\left(\partial G_{3}^{\prime} / \partial y_{1}^{\prime}\right) \xi_{1}^{\prime} \\
& +\left(\partial G_{3}^{\prime} / \partial y_{3}\right) \int_{x_{0}}^{x} e^{V_{2}}\left[\left(\partial G_{3}^{\prime} / \partial y_{1}\right) \xi_{1}+\left(\partial G_{3}^{\prime} / \partial y_{1}^{\prime}\right) \xi_{1}^{\prime}\right] d t .
\end{aligned}
$$

When the values of $\theta_{a}$ and $\theta_{a}^{\prime}$ as given by (5) and (6) are substituted in the expression defining the partial variation of $I_{1}$ with respect to $a$, it becomes for $a=b=0$

$$
\begin{aligned}
\left(\partial I_{1} / \partial a\right) \delta a=\int_{x_{0}}^{x_{1}}[ & {\left[\partial F_{1} / \partial y_{1}+\left(\partial F_{1} / \partial y_{3}^{\prime}\right) \partial G_{3}^{\prime} / \partial y_{1}\right] \xi_{1}+\left[\left(\partial F_{1} / \partial y_{3}^{\prime}\right) \partial G_{3}^{\prime} / \partial y_{1}^{\prime}\right.} \\
\left.+\partial F_{1} / \partial y_{1}^{\prime}\right] \xi_{1}^{\prime} & +\left[\partial F_{1} / \partial y_{3}+\left(\partial F_{1} / \partial y_{3}^{\prime}\right) \partial G_{3}^{\prime} / \partial y_{3}\right] \int_{x_{0}}^{x} e^{V_{0}\left[\left(\partial G_{3}^{\prime} / \partial y_{1}\right) \xi_{1}\right.} \\
& \left.\left.+\left(\partial G_{3}^{\prime} / \partial y_{1}^{\prime}\right) \xi_{1}^{\prime}\right] d t\right] d x .
\end{aligned}
$$

An application of Dirichlet's formula for changing the order of integration of an iterated integral, followed by an interchange of $t$ and $x$, the parameters of integration, yields the equation

\footnotetext{
* Hadamard, loc. cit., Chapter VI, gives the classical theory.
} 


$$
\begin{aligned}
\left(\partial I_{1} / \partial a\right) \delta a= & \int_{x_{0}}^{x_{1}}\left[\left[\partial F_{1} / \partial y_{1}+\left(\partial F_{1} / \partial y_{3}^{\prime}\right) \partial G_{3}^{\prime} / \partial y_{1}+\left(\partial G_{3}^{\prime} / \partial y_{1}\right) W_{1}\right] \xi_{1}\right. \\
& \left.+\left[\partial F_{1} / \partial y_{1}^{\prime}+\left(\partial F_{1} / \partial y_{3}^{\prime}\right) \partial G_{3}^{\prime} / \partial y_{1}^{\prime}+\left(\partial G_{3}^{\prime} / \partial y_{1}^{\prime}\right) W_{1}\right] \xi_{1}^{\prime}\right] d x
\end{aligned}
$$

where

$$
W_{1}=\int_{x}^{x_{1}} e^{V_{3}}\left[\partial F_{1} / \partial y_{3}+\left(\partial F_{1} / \partial y_{3}^{\prime}\right) \partial G_{3}^{\prime} / \partial y_{3}\right] d t
$$

Since $\xi_{1}(x)$ vanishes at $x_{0}$ and $x_{1}$ by hypothesis, an integration by parts performed on the terms involving $\xi_{1}(x)$ of the partial variation of $I_{1}$ with respect to $a$ furnishes the expression

$$
\begin{aligned}
\left(\partial I_{1} / \partial a\right) \delta a= & \int_{x_{0}}^{x_{1}}\left[-\int_{x_{0}}^{x}\left[\partial F_{1} / \partial y_{1}+\left(\partial F_{1} / \partial y_{3}^{\prime}\right) \partial G_{3}^{\prime} / \partial y_{1}+\left(\partial G_{3}^{\prime} / \partial y_{1}\right) W_{1}\right] d t\right. \\
& \left.+\left[\partial F_{1} / \partial y_{1}^{\prime}+\left(\partial F_{1} / \partial y_{3}^{\prime}\right) \partial G_{3}^{\prime} / \partial y_{1}^{\prime}+\left(\partial G_{3}^{\prime} / \partial y_{1}^{\prime}\right) W_{1}\right]\right] \xi_{1}^{\prime}(x) d x
\end{aligned}
$$

where the coefficient of $\xi_{1}^{\prime}(x)$ is continuous because of the continuity requirements on $F_{1}$ and $G$.

If $I_{1}$ is to be a maximum along the curve $\Gamma$, it is necessary that $\left(\delta I_{1} / \partial a\right) \delta a$ be zero for all values of the functions $\xi_{1}(x)$. By a well known theorem of the calculus of variations it follows that the coefficient of $\xi_{1}^{\prime}(x)$ must be a constant, that is,

$$
\begin{aligned}
& \partial F_{1} / \partial y_{1}^{\prime}+\left(\partial F_{1} / \partial y_{3}^{\prime}\right) \partial G_{3}^{\prime} / \partial y_{1}^{\prime}+\left(\partial G_{3}^{\prime} / \partial y_{1}^{\prime}\right) W_{1} \\
& =\int_{x_{0}}^{x}\left[\partial F_{1} / \partial y_{1}+\left(\partial F_{1} / \partial y_{3}^{\prime}\right) \partial G_{3}^{\prime} / \partial y_{1}+\left(\partial G_{3}^{\prime} / \partial y_{1}\right) W_{1}\right] d t+C_{1},
\end{aligned}
$$

where $C_{1}$ is a constant to be determined by the initial conditions.

An entirely similar analysis applied to $I_{2}$ yields the necessary condition

$$
\begin{gathered}
\partial F_{2} / \partial y_{2}^{\prime}+\left(\partial F_{2} / \partial y_{3}^{\prime}\right) \partial G_{3}^{\prime} / \partial y_{2}^{\prime}+\left(\partial G_{3}^{\prime} / \partial y_{2}^{\prime}\right) W_{2} \\
=\int_{x_{0}}^{x}\left[\partial F_{2} / \partial y_{2}+\left(\partial F_{2} / \partial y_{3}^{\prime}\right) \partial G_{3}^{\prime} / \partial y_{2}+\left(\partial G_{3}^{\prime} / \partial y_{2}\right) W_{2}\right] d t+C_{2} .
\end{gathered}
$$

The functional-differential equations (7) and (8) are the analogues of the Euler equations in the Du Bois-Reymond form.* Wherever the maximizing curve $\Gamma$ has a continuously turning tangent we can differentiate (7) and (8) with respect to $x$ and obtain functional-differential equations which involve

* Du Bois-Reymond, Mathematische Annalen. vol. 15 (1879), p. 313. 
second-order derivatives and which are the analogues of the Euler equations. We can, therefore, state the following theorem.

THEOREM 1. In order that an admissible arc $\Gamma$ in the space $\left(u_{1}, u_{2}, u_{3}, x\right)$, satisfying a differential equation $G\left(u_{1}, u_{1}^{\prime}, u_{2}, u_{2}^{\prime}, u_{3}, u_{3}^{\prime}, x\right)=0$ and initial conditions $u_{i}\left(x_{0}\right)=u_{i 0}, u_{i}\left(x_{1}\right)=u_{i 1}$, maximize an integral $I_{1}$ when $u_{2}$ is not allowed to vary and at the same time maximize a second integral $I_{2}$ when $u_{1}$ is not allowed to vary, it is necessary that this curve satisfy the functional-differential equations (7) and (8). If the maximizing curve has a continuously turning tangent at $x, x_{0} \leqq x \leqq x_{1}$, it must satisfy the equations

$$
\begin{aligned}
\partial F_{k} / \partial y_{k}+ & \left(\partial F_{k} / \partial y_{3}^{\prime}\right) \partial G_{3}^{\prime} / \partial y_{k}+\left(\partial G_{3}^{\prime} / \partial y_{k}\right) W_{k}-\frac{d}{d x}\left[\partial F_{k} / \partial y_{k}^{\prime}\right. \\
& \left.+\left(\partial F_{k} / \partial y_{3}^{\prime}\right) \partial G_{3}^{\prime} / \partial y_{k}^{\prime}+\left(\partial G_{3}^{\prime} / \partial y_{k}^{\prime}\right) W_{k}\right]=0 \quad(k=1,2),
\end{aligned}
$$

obtained by differentiating (7) and (8) with respect to $x$.

Functional-differential equations of the type (9), with our form of $W_{k}$, have not been discussed in the literature. It would be desirable to be able to say that a unique solution of these equations plus the differential equation $G=0$ exists whenever $t$ nd values $u_{i}\left(x_{0}\right)=u_{i 0}$ and $u_{i}\left(x_{1}\right)=u_{i 1}$ are given. This problem will not be discussed in the present paper.* It may be mentioned, however, that I have already exhibited a special example for which the system (9) reduces to a system of Volterra integral equations, and have actually found the solution. $\dagger$ Let us examine (7) and (8) from a different point of view.

In particular if $F_{1} \equiv F_{2}$, the problem reduces to a strict Lagrange problem. No assumption which would prevent this has been made, hence we have the following

CoRollary. The equations resulting from (7) and (8) by putting $F_{1} \equiv F_{2}$ must be satisfied by a curve satisfying a differential equation $G=0$ and initial conditions $u_{i}\left(x_{0}\right)=u_{i 0}, u_{i}\left(x_{1}\right)=u_{i 1}$ if this curve is to maximize an integral

$$
I=\int_{x_{0}}^{x_{1}} F_{1}\left(u_{1}, u_{1}^{\prime}, u_{2}, u_{2}^{\prime}, u_{3}, u_{3}^{\prime}, x\right) d x
$$

in which both $u_{1}$ and $u_{2}$ vary independently.

* L. M. Graves, Implicit functions and differential equations in general analysis, these Transactions, vol. 29, pp. 515-552, gives imbedding and existence theorems for a system which includes (9) as a special case. If $y_{k}{ }^{\prime \prime \prime}$ is continuous, we can reduce (9) to a differential equation of the third order by a differentiation, because of the form of $W_{k}$, and existence theorems for differential equations will apply.

† Roos, $A$ mathematical theory of competition, loc. cit., p. 167. 
The methods of this part can be extended without difficulty to the case for which there are $n$ integrals

$$
I_{h}=\int_{x_{0}}^{x_{1}} F_{h}\left(u_{1}, u_{1}^{\prime}, \cdots, u_{n}, u_{n}^{\prime}, u_{n+1}, u_{n+1}^{\prime}, x\right) d x \quad(h=1,2, \cdots, n)
$$

and one differential equation $G\left(u_{1}, u_{1}^{\prime}, \cdots, u_{n}, u_{n}^{\prime}, u_{n+1}, u_{n+1}^{\prime}, x\right)=0$, in which case $n$ functional equations of the type (7) result.

\section{Variable END poInts. ANalogues of Weierstrass and Legendre CONDITIONS}

4. Problem with one end point variable. In the preceding paragraphs a problem in simultaneous maxima for fixed end points has been considered. The problem is even more interesting when one end parameter, say $x_{1}$, and the corresponding end values are allowed to vary.

Consider the problem of determining a curve $\Gamma$ in the space $\left(u_{1}, u_{2}, u_{3}\right.$, $\left.u_{4}, x\right)$ satisfying a differential equation

$$
G\left(u_{1}, u_{1}^{\prime}, u_{2}, u_{2}^{\prime}, u_{3}, u_{3}^{\prime}, u_{4}, u_{4}^{\prime}, x\right)=0
$$

such that an integral

$$
I_{1}=\int_{x_{0}}^{x_{1}} F\left(u_{1}, u_{1}^{\prime}, \cdots, u_{4}, u_{4}^{\prime}, x\right) d x
$$

is a maximum when $u_{1}$ and $u_{2}$ are allowed to vary independently, but not $u_{3}$, and such that a second integral

$$
I_{2}=\int_{x_{0}}^{x_{1}} F_{2}\left(u_{1}, u_{1}^{\prime}, \cdots, u_{4}, u_{4}^{\prime}, x\right) d x
$$

is a maximum when $u_{3}$ is allowed to vary independently, but not $u_{1}$ and $u_{2}$. We assume the end parameter $x_{0}$ and the end values $u_{i}\left(x_{0}\right)=u_{i 0}$ to be fixed, and the end parameter $x_{1}$ and the corresponding end values of the $u_{i}$ to be variable. Let us assume as we did in Part II that $\partial G / \partial u_{4}^{\prime} \neq 0$ for the region which contains admissible arcs $u_{i}(x), i=1,2,3,4$, and that the functions $F_{\alpha}, \alpha=1,2$, and $G$ are continuous in $u_{1}, u_{2}, u_{3}, u_{4}, u_{1}^{\prime}, u_{2}^{\prime}, u_{3}^{\prime}, u_{4}^{\prime}, x$ and have continuous partial derivatives of the first order with respect to these arguments.

5. Functional transversality conditions. In the functions $F_{\alpha}$ and $G$ replace the functions $u_{i}(x), i=1,2,3,4$, by a set $u_{i}=f_{i}\left(x, a_{1}, a_{2}, a_{3}\right)$, where the $f_{i}$ are functions of $x$ and parameters $a_{1}, a_{2}$ and $a_{3}$, continuous and admitting continuous derivatives up to the second order with respect to $x$ and these parameters in the domain $0 \leqq a_{1} \leqq h ; 0 \leqq a_{2} \leqq h ; 0 \leqq a_{3} \leqq h ; x_{0} \leqq x \leqq x_{1}$. The 
functions $f_{1}, f_{2}$ and $f_{3}$ are otherwise arbitrary, but $f_{4}$ is determined by $G=0$ and the initial condition $u_{4}\left(x_{0}\right)=u_{40}$. Let the limit of integration $x_{1}$ be a similar function of the parameters $a_{\sigma}, \sigma=1,2,3$, i.e. $x_{1}=\phi\left(a_{1}, a_{2}, a_{3}\right)$.

By the ordinary rules of differentiation the differential of the integral $I_{1}$, which is also a function of the $a_{\sigma}$, is for $u_{3}$ (=constant)

$$
\begin{aligned}
d I_{1}=\left[F _ { 1 } \left(u_{1}, u_{1}^{\prime}, \cdots,\right.\right. & \left.\left.u_{4}, u_{4}^{\prime}, x\right) \delta x\right]^{x_{1}} \\
& +\int_{x_{0}}^{x_{1}}\left[\left(\partial F_{1} / \partial u_{i}\right) \delta f_{i}+\left(\partial F_{1} / \partial u_{i}^{\prime}\right) \delta f_{i}^{\prime}\right] d x,
\end{aligned}
$$

where $\delta f_{i}=\left(\partial F_{i} / \partial a_{1}\right) \delta a_{1}+\left(\partial f_{i} / \partial a_{2}\right) \delta a_{2}+\left(\partial f_{i} / \partial a_{3}\right) \delta a_{3}$ and $i$ is an umbral index for the values $1,2,4$, but not for 3 , according to the convention that whenever a literal suffix appears twice in a term that term is to be summed for values of the suffix.* The variation of $u_{3}$ in $F_{1}$ is by hypothesis equal to zero, hence $\delta f_{3} \equiv 0$.

As already stated the variations of $f_{1}$ and $f_{2}$ are to be arbitrary (except for continuity properties), but we can not take the variation of $f_{4}$ to be arbitrary, for it is determined by the differential equation of partial variation

$$
\left(\partial G / \partial u_{4}\right) \delta f_{4}+\left(\partial G / \partial u_{4}^{\prime}\right) \delta f_{4}^{\prime}+\left(\partial G / \partial u_{k}\right) \delta f_{k}+\left(\partial G / \partial u_{k}^{\prime}\right) \delta f_{k}^{\prime}=0,
$$

where $k$ is an umbral index taking on the values 1 and 2 only. Since $\partial G / \partial u_{i}^{\prime}$ does not vanish and is continuous in the interval by hypothesis, and, furthermore, since $\delta(d f / d x)=(d / d x) \delta f$, this expression can be regarded as a firstorder differential equation for the determination of $\delta f_{4}$ in terms of $\delta f_{1}$ and $\delta f_{2}$ and the initial value of $\delta f_{4}$ at $x=x_{0}$. Since we have supposed $\delta f_{4}\left(x_{0}\right)=0$, we may write

$$
\delta f_{4}=\int_{x_{0}}^{x} e^{v_{4}}\left[\left(\partial G_{4}^{\prime} / \partial u_{k}\right) \delta f_{k}+\left(\partial G_{4}^{\prime} / \partial u_{k}^{\prime}\right) \delta f_{k}^{\prime}\right] d t,
$$

where the expressions of the form $\partial G_{4}^{\prime} / \partial u_{k}$, etc. have meanings similar to the corresponding ratios defined in (5). As in (5) we determine the value of $\delta f_{4}^{\prime}$ by differentiation of the above expression. If the values of $\delta f_{4}$ and $\delta f_{4}^{\prime}$ so found be substituted in (10), it becomes

$$
\begin{gathered}
\left.F_{1} \delta x\right]^{x_{1}}+\int_{x_{0}}^{x_{1}}\left[\left[\partial F_{1} / \partial u_{k}+\left(\partial F_{1} / \partial u_{4}^{\prime}\right)\left(\partial G_{4}^{\prime} / \partial u_{k}\right)\right] \delta f_{k}\right. \\
+\left[\partial F_{1} / \partial u_{k}^{\prime}+\left(\partial F_{1} / \partial u_{4}^{\prime}\right)\left(\partial G_{4}^{\prime} / \partial u_{k}^{\prime}\right)\right] \delta f_{k}^{\prime} \\
\left.+\left[\partial F_{1} / \partial u_{4}+\left(\partial F_{1} / \partial u_{4}^{\prime}\right)\left(\partial G_{4}^{\prime} / \partial u_{4}\right)\right] \int_{x_{0}}^{x} e^{V} \cdot\left[\left(\partial G_{4}^{\prime} / \partial u_{k}\right) \delta f_{k}+\left(\partial G_{4}^{\prime} / \partial u_{k}^{\prime}\right) \delta f_{k}^{\prime}\right] d t\right] d x .
\end{gathered}
$$

* See A. S. Eddington, The Mathematical Theory of Relativity, p. 50. 
An application of Dirichlet's formula to the iterated integral followed by an interchange of the parameters $x$ and $t$ as before reduces the above formula for $\delta I_{1}$ to

$$
\begin{gathered}
\left.\delta I_{1}=F_{1} \delta x\right]^{x_{1}}+\int_{x_{0}}^{x_{1}}\left[\left[\partial F_{1} / \partial u_{k}+\left(\partial F_{1} / \partial u_{4}^{\prime}\right)\left(\partial G_{4}^{\prime} / \partial u_{k}\right)\right.\right. \\
\left.+\left(\partial G_{4}^{\prime} / \partial u_{k}\right) W_{1}\right] \delta f_{k}+\left[\partial F_{1} / \partial u_{k}^{\prime}+\left(\partial F_{1} / \partial u_{4}^{\prime}\right)\left(\partial G_{4}^{\prime} / \partial u_{k}^{\prime}\right)\right. \\
\left.\left.+\left(\partial G_{4}^{\prime} / \partial u_{k}^{\prime}\right) W_{1}\right] \delta f_{k}^{\prime}\right] d x
\end{gathered}
$$

where

$$
W_{1}=\int_{x_{1}}^{x} e^{\nabla} \cdot\left[\left(\partial F_{1} / \partial u_{4}^{\prime}\right)\left(\partial G_{4}^{\prime} / \partial u_{4}\right)+\partial F_{1} / \partial u_{4}\right] d t .
$$

Since the $f_{k}\left(x, a_{1}, a_{2}, a_{3}\right), k=1,2$, have by hypothesis continuous second derivatives with respect to $x$, the formula for integration by parts can be applied to the second member of (10B), so that*

$$
\begin{gathered}
\delta I_{1}=\left[F_{1} \delta x+\left[\partial F_{1} / \partial u_{k}^{\prime}+\left(\partial F_{1} / \partial u_{4}^{\prime}\right)\left(\partial G_{i}^{\prime} / \partial u_{k}^{\prime}\right)+\left(\partial G_{4}^{\prime} / \partial u_{k}^{\prime}\right) W_{1}\right] \delta f_{k}\right]_{x_{0}}^{x_{1}} \\
+\int_{x_{0}}^{x_{1}}\left[\partial F_{1} / \partial u_{k}+\left(\partial F_{1} / \partial u_{4}^{\prime}\right)\left(\partial G_{4}^{\prime} / \partial u_{k}\right)+\left(\partial G_{4}^{\prime} / \partial u_{k}\right) W_{1}\right. \\
\left.-\frac{d}{d x}\left[\partial F_{1} / \partial u_{k}^{\prime}+\left(\partial F_{1} / \partial u_{4}^{\prime}\right)\left(\partial G_{4}^{\prime} / \partial u_{k}^{\prime}\right)+\left(\partial G_{4}^{\prime} / \partial u_{k}^{\prime}\right) W_{1}\right]\right] \delta f_{k} d x
\end{gathered}
$$

where $k$ is umbral as before.

By definition $\delta f_{k}=\left(\partial F_{k} / \partial a_{i}\right) \delta a_{i}$, where $i$ is umbral with range $1,2,3$, hence the variation of $u_{k}$ is given by $\delta u_{k}=u_{k}^{\prime} \delta x+\delta f_{k}$. If the value of $\delta f_{k}$ defined by this equation be substituted in $\delta I_{1}$, the following formula results:

$$
\begin{aligned}
\delta I_{1}= & {\left[\partial F_{1} / \partial u_{k}^{\prime}+\left[\left(\partial F_{1} / \partial u_{4}^{\prime}\right)\left(\partial G_{4}^{\prime} / \partial u_{k}^{\prime}\right)+\left(\partial G_{4}^{\prime} / \partial u_{k}^{\prime}\right) W_{1}\right]\left[\delta u_{k}-u_{k}^{\prime} \delta x\right]\right]_{2_{0}}^{x_{1}} } \\
& +\int_{x_{0}}^{x_{1}}\left[\partial F_{1} / \partial u_{k}+\left(\partial F_{1} / \partial u_{4}^{\prime}\right)\left(\partial G_{4}^{\prime} / \partial u_{k}\right)+\left(\partial G_{4}^{\prime} / \partial u_{k}\right) W_{1}\right. \\
& \left.-\frac{d}{d x}\left[\partial F_{1} / \partial u_{k}^{\prime}+\left(\partial F_{1} / \partial u_{4}^{\prime}\right)\left(\partial G_{4}^{\prime} / \partial u_{k}^{\prime}\right)+\left(\partial G_{4}^{\prime} / \partial u_{k}^{\prime}\right) W_{1}\right]\right] \delta f_{k} d x .
\end{aligned}
$$

We define an arc $u_{i}=u_{i}(x), i=1,2,3,4$, as an extremal arc if it has continuous derivatives $d u_{i} / d x$ and $d^{2} u_{i} / d x^{2}$ in the interval $x_{0} \leqq x \leqq x_{1}$, and if, furthermore, it satisfies the differential equation $G\left(u_{1}, u_{1}^{\prime}, \cdots, u_{4}, u_{4}^{\prime}, x\right)=0$, the set of two equations

\footnotetext{
* Hadamard, loc cit., p. 60.
} 


$$
\begin{aligned}
& \partial F_{1} / \partial u_{k}+\left(\partial F_{1} / \partial u_{4}^{\prime}\right)\left(\partial G_{4}^{\prime} / \partial u_{k}\right)+\left(\partial G_{4}^{\prime} / \partial u_{k}\right) W_{1}-\frac{d}{d x}\left[\partial F_{1} / \partial u_{k}^{\prime}\right. \\
& \left.+\left(\partial F_{1} / \partial u_{4}^{\prime}\right)\left(\partial G_{4}^{\prime} / \partial u_{k}^{\prime}\right)+\left(\partial G_{4}^{\prime} / \partial u_{k}^{\prime}\right) W_{1}\right]=0 \quad(k=1,2),
\end{aligned}
$$

and a similar set for the integral $I_{2}$.

If an extremal $\Gamma$ is to maximize $I_{1}$ for $u_{3}$ constant, it is necessary that the differential

$$
\delta I_{1}(\Gamma)=F_{1} \delta x_{1}+\left[\partial F_{1} / \partial u_{k}^{\prime}+\left(\partial F_{1} / \partial u_{4}^{\prime}\right) \partial G_{4}^{\prime} / \partial u_{k}^{\prime}\right]\left[\delta u_{k}\left(x_{1}\right)-u_{k}^{\prime} \delta x_{1}\right]
$$

vanish for all possible choices of $\delta x_{1}$ and $\delta u_{k}\left(x_{1}\right), k=1,2$. We can thus state the transversality theorem:

THEOREM 2. If for an admissible arc $\Gamma$, one of whose end points is fixed at $x_{0}$ while the other varies over $a V_{3}$ defined by $u_{3}=$ constant and a differential equation $G=0$, the value $I_{1}(\Gamma)$, for $u_{3}=$ constant, $G=0$, is a maximum with respect to the values of $I_{1}$ on neighboring admissible arcs, issuing from the same fixed point 0 , then at the intersection point 1 of $\Gamma$ with $V_{3}$, the directional coefficients of $V_{3}$ and the element $\left(u_{1}, u_{1}^{\prime}, \cdots, u_{4}^{\prime}, x\right)$ of $\Gamma$ must satisfy the relations

$$
\begin{aligned}
& F_{1}\left(u_{1}, u_{1}^{\prime}, \cdots, u_{4}^{\prime}, x\right)-\left[\partial F_{1} / \partial u_{k}^{\prime}+\left(\partial F_{1} / \partial u_{4}^{\prime}\right)\left(\partial G_{4}^{\prime} / \partial u_{k}^{\prime}\right)\right] u_{k}^{\prime}=0 \\
& \partial F_{1} / \partial u_{i}^{\prime}+\left(\partial F_{1} / \partial u_{4}^{\prime}\right)\left(\partial G_{4}^{\prime} / \partial u_{i}^{\prime}\right)=0^{*} \\
& (i=1,2) .
\end{aligned}
$$

If we apply a similar analysis to the integral $I_{2}$, for $u_{1}$ and $u_{2}$ constant, we obtain a differential

$$
\delta I_{2}(\Gamma)=F_{2} \delta x_{1}+\left[\partial F_{2} / \partial u_{3}^{\prime}+\left(\partial F_{2} / \partial u_{3}^{\prime}\right)\left(\partial G_{4}^{\prime} / \partial u_{3}{ }^{\prime}\right)\right]\left[\delta u_{3}\left(x_{1}\right)-u_{3}^{\prime}\left(x_{1}\right) \delta x_{1}\right]
$$

along an extremal for the integral $I_{2}$. If, therefore, $\Gamma$ is also to maximize $I_{2}$ for $u_{1}$ and $u_{2}$ constant, then at 1 , the intersection of $\Gamma$ with $V_{2}$, defined by $u_{1}=$ constant, $u_{2}=$ constant and $G=0$, it is necessary that the equations

$$
\begin{aligned}
& F_{2}\left(u_{1}, u_{1}^{\prime}, \cdots, u_{4}^{\prime}, x\right)+\left[\partial F_{2} / \partial u_{3}^{\prime}+\left(\partial F_{2} / \partial u_{4}^{\prime}\right)\left(\partial G_{4}^{\prime} / \partial u_{3}^{\prime}\right)\right] u_{3}^{\prime}=0, \\
& \partial F_{2} / \partial u_{3}^{\prime}+\left(\partial F_{2} / \partial u_{4}^{\prime}\right)\left(\partial G_{4}^{\prime} / \partial u_{3}^{\prime}\right)=0
\end{aligned}
$$

* From a consideration of the classical theory of the Lagrange problem with second end point variable we would expect to have four transversality conditions instead of three as given by (12), but we have not used the condition that $\delta u_{4}\left(x_{1}\right)$ is arbitrary, since it is a function of arbitrary functions $\delta f_{k}$, and hence we lack this condition. If we perform an integration by parts on the term in $\delta f_{i}^{\prime}$ of $(10)$, and then substitute for $\delta f_{i}$ as we did above, we obtain a term $\left(\partial F_{1} / \partial u_{4}{ }^{\prime}\right) \delta f_{4}$ besides terms in $\delta f_{k}$. Since $\delta f_{4}$ is arbitrary at $x_{1}$ it follows that $\partial F_{1} / \partial u_{4}^{\prime}=0$ at $x=x_{1}$. We may, therefore, by the help of (12) write the transversality condition as

$$
\begin{aligned}
& F_{1}\left(u_{1}, u_{1}^{\prime}, \cdots, u_{4}^{\prime}, x\right)-\left(\partial F_{1} / \partial u_{k}{ }^{\prime}\right) u_{k}{ }^{\prime}=0, \\
& \partial F_{1} / \partial u_{k}^{\prime}=0 \\
& (k=1,2,4) .
\end{aligned}
$$

The equations (12A) are the analogues of the usual transversality conditions. (See Bliss, loc. cit., p. 167.) 
hold. In equations (12) and (13) we have five equations for the determination of the four end values $x_{1}, u_{h}\left(x_{1}\right), h=1,2,3$, of an extrc:nal $\Gamma$. In general, therefore, the problem of simultaneous maxima is not possible for the case for which the second end parameter $x_{1}$ is required to be the same for both $I_{1}$ and $I_{2}$. Hence, it will be understood in our work that $x_{1}$ has, in general, different values for $I_{1}$ and $I_{2}$. The conditions (12) and (13) are functional in form and will be called functional transversality conditions.

6. Analogue of the Weierstrass necessary condition. By the aid of the expression for $\delta I_{1}(\Gamma)$ we can state the following theorem:

TheOREM 3. The value of an integral $I_{1}$, taken along a two-parameter family of extremal arcs $E_{01}$ determined by the equations $u_{k}=f_{k}\left(x, a_{1}, a_{2}\right), k=1,2,4$, $G=0$, and the hypersurface $u_{3}=f_{3}(x)=$ constant, one of whose end points, $x_{0}$, is fixed while the other, $x_{1}$, varies, has a differential

$$
\begin{aligned}
d I_{1}=F_{1}\left(u_{1}, p_{1}, u_{2}, p_{2}, u_{3}, u_{3}^{\prime}, u_{4}, p_{4}, x\right) d x_{1} \\
+\left[\partial F_{1} / \partial u_{k}^{\prime}+\left(\partial F_{1} / \partial u_{4}^{\prime}\right)\left(\partial G_{4}^{\prime} / \partial u_{k}^{\prime}\right)\right]\left[d u_{k}\left(x_{1}\right)-p_{k} d x_{1}\right],
\end{aligned}
$$

where at the point 1 , the differentials $d x_{1}$ and $d u_{k}$ are those belonging to $V_{3}\left(u_{3}=\right.$ constant) described by the end points of the extremals, while the $u_{i}, u_{3}, p_{i}$ and $u_{3}^{\prime}$ refer to the extremal $E_{01}$. The functions $F_{1}$ and $G$ have arguments $\left(u_{1}, p_{1}, u_{2}\right.$, $\left.p_{2}, u_{3}, u_{3}^{\prime}, u_{4}, p_{4}, x\right)$, where the $p_{1}, p_{2}$ and $p_{4}$ are the directional coefficients of the extremal $E_{01}$ for $u_{3}=$ constant.

There is an entirely analogous theorem for the integral $I_{2}$. For $I_{2}$ the functions $F_{2}$ and $G$ have arguments $\left(u_{1}, u_{1}^{\prime}, u_{2}, u_{2}^{\prime}, u_{3}, p_{3}, u_{4}, p_{4}, x\right)$.

The integral of $d I_{1}$ corresponds to the Hilbert integral and possesses similar properties. In a manner analogous to the classical method of the calculus of variations it is possible to obtain the necessary conditions of Weierstrass and Legendre and to obtain sufficient conditions for relative strong and weak maxima.* To do this we first define an extremal field in the sense in which we shall use it in this chapter.

We shall say that a connected region $R$ of the space $\left(u_{1}, u_{2}, u_{3}, u_{4}, x\right)$ is a simply covered extremal field if there exists a family of extremals dependent upon three parameters such that one and only one extremal of this simply covered field passes through every point of $R$, and if, furthermore, the directional coefficients $d u_{h} / d x=p_{h}\left(u_{1}, \cdots, u_{4}, x\right), h=1, \cdots, 4$, of the tangent to the extremal, which passes through the point $\left(u_{1}, u_{2}, u_{3}, u_{4}, x\right)$, are continuous functions, admitting continuous partial derivatives in $R$

* For the classical analysis see Hadamard, loc. cit., p. 364. See also Bliss, loc. cit., p. 50. 
up to the second order. We shall assume that such a field exists and that it contains $V_{3}$.

It is quite evident that along an extremal arc of a field, the integral $I_{1}{ }^{*}=\int d I_{1}$ has the same value as $I_{1}$, for $\delta u_{i}=p_{i} \delta x$ along an extremal, and the integrand of $I_{1}{ }^{*}$ thus reduces to the integrand of $I_{1}$.

To obtain an analogue of the Weierstrass condition we select a point (3) on $E_{01}$, the extremal which we are assuming to give the desired maximum, and through this point (3), holding $f_{3}\left(x, a_{1}, a_{2}, a_{3}\right)=u_{3}(x)$ constant, pass an otherwise arbitrary curve $C_{12}$ with continuously turning tangent in $R$. We note that $R$ may be partly bounded by $V_{3}$, so that when (3) is at (1) the curves $C_{12}$ are further limited. Such a curve $C_{12}$ will have equations $u_{1}=U_{1}(t)$, $u_{2}=U_{2}(t), u_{3}=u_{3}(t), u_{4}=U_{4}(t)$.

We join the fixed point 0 to a movable point 2 on $C_{12}$ by a one-parameter family of arcs $E_{02}$, containing $E_{01}$ as a member when the point 2 is in the position 1. We choose the parameter $t$ on $C_{21}$ increasing as 2 moves towards 1 , noting that the arc length $s$ is a possible $t$. If $E_{01}$ is to give a maximum for admissible arcs in $R$, i.e., $I_{1}\left(E_{02}+C_{21}\right) \leqq I_{1}\left(E_{01}\right)$, where $C_{12}$ and $E_{02}$ are obtained by putting $a_{3}=0$, it follows that $d I_{1}\left(E_{02}+C_{21}\right)=d I_{1}\left(E_{02}-C_{12}\right)$ must be $\geqq 0$ for 2 sufficiently close to 1 , and in particular at 1 itself, that is, $d I_{1}\left(C_{12}-E_{02}\right) \leqq 0$ must hold.

This differential is given by the value at the point 1 of the expression

$$
\begin{aligned}
F_{1}\left(U_{1},\right. & \left.U_{1}^{\prime}, U_{2}, U_{2}^{\prime}, u_{3}, u_{3}^{\prime}, U_{4}, U_{4}^{\prime}\right) \delta x \\
& -F_{1}\left(u_{1}, u_{1}^{\prime}, u_{2}, u_{2}^{\prime}, u_{3}, u_{3}^{\prime}, u_{4}, u_{4}^{\prime}, x\right) \delta x \\
& -\left[\partial F_{1} / \partial u_{k}^{\prime}+\left(\partial F_{1} / \partial u_{4}^{\prime}\right)\left(\partial G_{4}^{\prime} / \partial u_{k}^{\prime}\right)\right]\left[\delta u_{k}-u_{k}^{\prime} \delta x\right],
\end{aligned}
$$

the differentials in this expression belonging to the arc $C_{12}$ and, therefore, satisfying the equation $\delta u_{k}=U_{k}^{\prime} \delta x$. At the point 1 the coördinates of $C_{12}$ and $E_{02}$ are equal, so that this expression can be written as

$$
\begin{aligned}
d I_{1}\left(C_{12}-E_{02}\right)= & {\left[F_{1}\left(u_{1}, U_{1}^{\prime}, u_{2}, U_{2}^{\prime}, u_{3}, u_{3}^{\prime}, u_{4}, U_{4}^{\prime}, x\right)\right.} \\
& -F_{1}\left(u_{1}, u_{1}^{\prime}, u_{2}, u_{2}^{\prime}, u_{3}, u_{3}^{\prime}, u_{4}, u_{4}^{\prime}, x\right) \\
& \left.-\left[U_{k}^{\prime}-u_{k}^{\prime}\right]\left[\partial F_{1} / \partial u_{k}^{\prime}+\left(\partial F_{1} / \partial u_{4}^{\prime}\right)\left(\partial G_{4}^{\prime} / \partial u^{\prime}\right)\right]\right] \delta x .
\end{aligned}
$$

We shall call the coefficient of $\delta x$ in the above expression $E_{1}$, because it is an analogue of the Weierstrass $E$-function. $\dagger$ Since the differential $d I_{1}\left(C_{12}-E_{01}\right)$ must be negative or zero for an arbitrarily selected point 1 and an $\operatorname{arc} C$ through it, we have the following theorem:

† Bliss, loc. cit., p. 130. 
Theorem 4. At every element $\left(u_{1}, u_{1}^{\prime}, \cdots, u_{4}, u_{4}^{\prime}, x\right)$ of an arc $E_{01}$ which maximizes an integral $I_{3}$ when $u_{3}$ is not allowed to vary and which satisfies a differential equation $G\left(u_{1}, u_{1}^{\prime}, \cdots, u_{4}, u_{4}^{\prime}, x\right)=0$, the Weierstrass condition

$$
E_{1}\left(u_{1}, u_{1}^{\prime}, U_{1}^{\prime}, u_{2}, u_{2}^{\prime}, U_{2}^{\prime}, u_{3}, u_{3}^{\prime}, u_{4}, u_{4}^{\prime}, U_{4}^{\prime}, x\right) \leqq 0
$$

must be satisfied for every admissible set $\left(u_{1}^{\prime}, U_{1}, u_{2}, U_{2}^{\prime}, u_{3}, u_{3}^{\prime}, u_{4}, U_{4}^{\prime}\right)$, different from $\left(u_{1}, u_{1}^{\prime}, u_{2}, u_{2}^{\prime}, u_{3}, u_{3}^{\prime}, u_{4}, u_{4}^{\prime}, x\right)$, for all values of the coördinates $\left(u_{1}, u_{2}, u_{3}, u_{4}, x\right)$ in the region $R$.

A similar analysis applied to the integral $I_{2}$ yields the following theorem:

Theorem 5. At every element $\left(u_{1}, u_{1}^{\prime}, \cdots, u_{4}, u_{4}^{\prime}, x\right)$ of an arc $E_{01}$ which maximizes an integral $I_{2}$ when $u_{1}$ and $u_{2}$ are not allowed to vary and which satisfies a differential equation, $G=0$, the condition

$$
E_{2}\left(u_{1}, u_{1}^{\prime}, u_{2}, u_{2}^{\prime}, u_{3}, u_{3}^{\prime}, U_{3}^{\prime}, u_{4}^{\prime}, u_{4}^{\prime}, U_{4}^{\prime}, x\right) \leqq 0
$$

must be satisfied for every admissible set $\left(u_{1}, u_{1}^{\prime}, u_{2}, u_{2}^{\prime}, u_{3}, U_{3}^{\prime}, u_{4}, U_{4}^{\prime}, x\right)$ different from $\left(u_{1}, u_{1}^{\prime}, u_{2}, u_{2}^{\prime}, u_{3}, u_{3}^{\prime}, u_{4}, u_{4}^{\prime}, x\right)$, for all values of the coördinates $\left(u_{1}, u_{2}, u_{3}, u_{4}, x\right)$ in the region $R$.

The conditions (11), (12), (13), (14) and (15) are necessary conditions which must be satisfied by an $\operatorname{arc} E_{01}$ which furnishes a solution of the problem of this paper. In the following paragraph another necessary condition will be obtained.

7. Analogue of the Legendre necessary condition. For brevity we will consider only the first integral $I_{1}$. If the function $F_{1}\left(u_{1}, U_{1}^{\prime}, u_{2}, U_{2}^{\prime}, u_{3}, u_{3}^{\prime}\right.$, $\left.u_{4}, U_{4}^{\prime}, x\right)$ be expanded by means of Taylor's formula, the following expression is obtained:

$$
\begin{gathered}
F_{1}\left(u_{1}, U_{1}^{\prime}, u_{2}, U_{2}^{\prime}, u_{3}, u_{3}^{\prime}, u_{4}, U_{4}^{\prime}, x\right)=F_{1}\left(u_{1}, U_{1}^{\prime}, u_{2}, U_{2}^{\prime}, u_{8}, u_{3}^{\prime}, u_{4}, U_{4}^{\prime}, x\right) \\
+\left[U_{k}^{\prime}-u_{k}^{\prime}\right]\left[\partial F_{1} / \partial u_{k}^{\prime}+\left(\partial F_{1} / \partial u_{4}^{\prime}\right)\left(\partial u_{4}^{\prime} / \partial u_{k}^{\prime}\right)\right] \\
+\frac{1}{2}\left[U_{k}^{\prime}-u_{k}^{\prime}\right]\left[U_{h}^{\prime}-u_{h}^{\prime}\right] \partial A_{1 h} / \partial u_{k}^{\prime},
\end{gathered}
$$

where $A_{1 h}=\partial F_{1} / \partial u_{h}^{\prime}+\left(\partial F_{1} / \partial u_{4}^{\prime}\right)\left(\partial u_{4}^{\prime} / \partial u_{h}^{\prime}\right)$ and $h$ and $k$ are umbral indices with range 1,2 . The arguments of $A_{1 h}$ are $\left(u_{1}, u_{1}^{\prime}+\theta\left(U_{1}^{\prime}-u_{1}^{\prime}\right)\right.$, $\left.u_{2}, u_{2}^{\prime}+\theta\left(U_{2}^{\prime}-u_{2}^{\prime}\right), u_{3}, u_{3}^{\prime}, u_{4}, u_{4}^{\prime}+\theta\left(U_{1}^{\prime}-u_{4}^{\prime}\right), x\right)$, where $0<\theta<1$. It should be noted that in this formula $u_{3}$ is not allowed to vary.

Since the partial derivative of $G$ with respect to $u_{k}$ determines $\partial u_{k}^{\prime} / \partial u_{k}^{\prime}$, the function $E_{1}$ is given by the formula

$$
E_{1}\left(u_{1}, u_{1}^{\prime}, U_{1}^{\prime}, \cdots, u_{4}, u_{4}^{\prime}, U_{4}^{\prime}, x\right)=\frac{1}{2}\left(U_{k}^{\prime}-u_{k}^{\prime}\right)\left(U_{h}^{\prime}-u_{h}^{\prime}\right)\left(\partial A_{1 h} / \partial u_{k}^{\prime}\right) .
$$

Let us write $T=U_{1}^{\prime}-u_{1}^{\prime}, W=U_{2}^{\prime}-u_{2}^{\prime}$ and $V=U_{3}^{\prime}-u_{3}^{\prime}$. We may then by the help of (14) state the following theorem: 
Theorem 6. If the extremal $E_{01}$ makes $I_{1}$ a maximum when $u_{3}$ is not allowed to vary, and at the same time makes $I_{2}$ a maximum when $u_{1}$ and $u_{2}$ are not allowed to vary, it is necessary that the quadratic differential forms

$$
\begin{gathered}
T^{2}\left[\partial A_{11} / \partial u_{1}^{\prime}\right]+T W\left[\partial A_{12} / \partial u_{1}^{\prime}+\partial A_{11} / \partial u_{2}^{\prime}\right]+W^{2}\left[\partial A_{12} / \partial u_{2}^{\prime}\right] \\
V^{2}\left[\partial A_{23} / \partial u_{3}^{\prime}\right]
\end{gathered}
$$

be definite negative forms for all systems of finite values of $u_{1}^{\prime}, u_{2}^{\prime}, u_{3}^{\prime}$ and $u_{1}^{\prime}$, when the point $\left(u_{1}, u_{2}, u_{3}, u_{4}, x\right)$ remains in the domain $R . \dagger$

In (16) $u_{3}$ is not allowed to vary and in (17) $u_{1}$ and $u_{2}$ are not allowed to vary. In particular if we let $U_{h}^{\prime}$ approach $u_{h}^{\prime}$ in (16) we obtain a condition analogous to the Legendre condition. $\ddagger$

8. The analogue of the Jacobi condition. We proceed now to determine an analogue of the Jacobi condition for the problem of simultaneous maxima. Let $E_{02}$ and $E_{03}$ be two extremals of a two-parameter family, $u_{3}=$ constant, $u_{4}\left(x_{0}\right)=u_{40}$, through the point 0 , and suppose that these extremals touch an envelope $N$ of the family at their end points 2 and 3 . Since the differential $d I_{1}$ of $\S 6$ is a total differential, $u_{3}$ being constant, the integral $I_{1}{ }^{*}=\int d I_{1}$ around a closed contour $C$ is zero. As already pointed out in $\$ 6, I_{1}{ }^{*}$ along an extremal is identically equal to $I_{1}$, hence

$$
I_{1}\left(E_{03}\right)-I_{1}\left(E_{02}\right)=I_{1}^{*}\left(N_{23}\right) .
$$

The differentials $d x, d u_{i}, i=1, \cdots, 4$, at a point of the envelope satisfy the equations $d u_{i}=p_{i} d x$ with the slope $p$ of the extremal tangent to $N$ at that point. It follows then that $I_{1}^{*}\left(N_{23}\right)$ is the same as $I_{1}\left(N_{28}\right)$; hence the following theorem:

THEOREM 7. If $E_{02}$ and $E_{03}$ are two members of a two-parameter family, $u_{3}=$ constant, of extremals through the fixed point 0 , and if these touch an envelope $N$ of the family at their end points 2 and 3, then the values of the integral $I_{1}$ along the arcs $E_{02}, E_{03}$ and $N_{32}$ satisfy the equation $I_{1}\left(E_{02}\right)+I_{1}\left(N_{32}\right)=I_{1}\left(E_{03}\right)$ for every position of the point 3 preceding 2 on $N$.

This theorem is the analogue of the envelope theorem of the calculus of variations. $\S$ We have also a similar theorem for the integral $I_{2}$, for $u_{1}$ and $u_{2}$ held constant.

† For definition of definite negative forms see M. Bôcher, Introduction to Higher Algebra, p. 150.

† Hadamard, loc. cit., p. 391.

\& Bliss, loc. cit., pp. 140-141. 
Consider now the value of $I_{1}^{\prime}(0)=\delta I_{1}(0)$ given by equation (10B) for all end values fixed. Let $\lambda_{k}\left(u_{1}, u_{1}^{\prime}, \cdots, u_{4}, u_{4}^{\prime}, x\right)$ and $\zeta_{k}\left(u_{1}, u_{1}^{\prime}, \cdots\right.$, $\left.u_{4}, u_{4}^{\prime}, x\right)$ be, respectively, the coefficients of $\delta f_{k}$ and $\delta f_{k}^{\prime}$ in this expression. In order to simplify the notation further let $\delta f_{k}=\xi_{k}$. We can then write $(10 \mathrm{~B})$ as

$$
I_{1}^{\prime}(0)=\int_{x_{0}}^{x_{1}}\left[\lambda_{k} \xi_{k}+\zeta_{k} \xi_{k}^{\prime}\right] d x,
$$

where $k$ is umbral and ranges over 1 and 2 only. By a differentiation of this expression we obtain

$$
\begin{gathered}
I_{1}^{\prime \prime}(0)=\int_{x_{0}}^{x_{1}}\left[\left(\partial \lambda_{k} / \partial u_{h}\right) \xi_{k} \xi_{h}+\left(\partial \lambda_{k} / \partial u_{h}^{\prime}\right) \xi_{h}^{\prime} \xi_{k}+\left(\partial \lambda_{k} / \partial u_{4}\right) \xi_{k} \delta u_{4}\right. \\
+\left(\partial \lambda_{k} / \partial u_{4}^{\prime}\right) \xi_{k} \delta u_{4}^{\prime}+\left(\partial \zeta_{k} / \partial u_{h}\right) \xi_{h} \xi_{k}^{\prime}+\left(\partial \zeta_{k} / \partial u_{h}^{\prime}\right) \xi_{k}^{\prime} \xi_{h}^{\prime} \\
\left.+\left(\partial \zeta_{k} / \partial u_{4}\right) \xi_{k}^{\prime} \delta u_{4}+\left(\partial \zeta_{k} / \partial u_{4}^{\prime}\right) \xi_{k}^{\prime} \delta u_{4}^{\prime}\right] d x
\end{gathered}
$$

since $\xi_{3}$ is zero by hypothesis. The variations $\delta u_{4}$ and $\delta u_{4}^{\prime}$ are to be determined by the equations of partial variation of $\S 5$. We could carry out the substitution and application of Dirichlet's formula as before, and obtain an equation analogous to Jacobi's differential equation.* As one can readily see, this equation would be functional-differential in form, and would, therefore, be extremely difficult to handle. Instead of attempting to derive Jacobi's condition rigorously by means of this equation, we shall content ourselves with using a simpler and less rigorous method. $\dagger$

According to the last theorem the value of $I_{1}$ along the composite arc $E_{03}+N_{32}+E_{21}$ is always the same as its value along $E_{02}$. Since $N_{32}$ is not an extremal, it can be replaced by an arc $C_{34}$ giving $I_{1}$ a larger value, and hence $I_{1}\left(E_{01}\right)$ cannot be a minimum. $\ddagger$ As a further necessary condition we must, therefore, demand that there be no point 2 conjugate to 0 between 0 and 1 on a maximizing $\operatorname{arc} E_{01}$ which is an extremal, with a condition analogous to the condition $\partial^{2} F_{1} / \partial y^{\prime} \partial y^{\prime} \neq 0$ everywhere on it.

\section{SUFFICIENT CONDITIONS FOR SIMULTANEOUS MAXIMA}

9. Relative strong and weak maxima. By definition an extremal curve $E_{01}$ furnishes a strong relative maximum for an integral $I_{1}$ when $u_{3}$ is not allowed to vary, if there exist a positive number $\epsilon$ such that the integral

* Bliss, loc. cit., p. 163.

† Bliss, loc. cit., p. 141.

† To prove this we need to know that the functional-differential equations (9) defining an extremal have a unique solution at an arbitrarily selected point and direction. 


$$
I_{1}=\int_{x_{\mathrm{c}}}^{x_{1}} F_{1}\left(u_{1}, u_{1}^{\prime}, \cdots, u_{4}, u_{4}^{\prime}, x\right) d x
$$

is greater than the integral

$I_{1}(w)=\int_{x_{0}}^{x_{1}} F_{1}\left[u_{1}+w_{1}(x), u_{1}^{\prime}+w_{1}^{\prime}(x), \cdots, u_{4}+w_{4}(x), u_{4}^{\prime}+w_{4}^{\prime}(x), x\right] d x$

for all possible forms of the functions $w_{\sigma}, \sigma=1, \cdots, 4$, of class (I) in the interval $x_{0} \leqq x \leqq x_{1}$, and satisfying the conditions*

$$
w_{\sigma}\left(x_{0}\right)=0 ;\left|w_{\sigma}(x)\right|<\epsilon ; x_{0} \leqq x \leqq x_{1} .
$$

When in addition to the conditions above the functions $w_{\sigma}(x)$ satisfy $\left|w_{\sigma}^{\prime}(x)\right|<\epsilon$ for $x_{0} \leqq x \leqq x_{1}$, an extremal curve $\left(u_{1}, u_{2}, u_{3}, u_{4}, x\right)$ furnishes a weak relative maximum. $\dagger$

10. Sufficient conditions for a maximum. By means of the definition of an extremal field of $\S 6$ and the definitions of strong and weak relative maxima of $\$ 9$ we are now in a position to write sufficient conditions for both strong and weak relative maxima.

THEOREM 8. If $E_{01}$ is an extremal arc and if the conditions (14) and (15) without the equality sign are satisfied at every element $\left(u_{1}, u_{1}^{\prime}, \cdots, u_{4}, u_{4}^{\prime}, x\right)$ in a neighborhood $R^{\prime}$, contained in $R$, of the corresponding elements of $E_{01}$ for every admissible set $\left(u_{1}, U_{1}^{\prime}, \cdots, u_{4}, U_{4}^{\prime}, x\right)$ such that in (14) the expressions $\left(U_{1}^{\prime}-u_{1}^{\prime}\right)$ and $\left(U_{2}^{\prime}-u_{2}^{\prime}\right)$ are not both zero and yet $U_{3}^{\prime}-u_{3}^{\prime} \equiv 0$, and, furthermore, in (15) $\left(U_{3}^{\prime}-u_{3}^{\prime}\right)$ is not zero and yet $U_{1}^{\prime}-u_{1}^{\prime} \equiv 0$ and $U_{2}^{\prime}-u_{2}^{\prime} \equiv 0$, and if, finally, there is no point 2 conjugate to 0 between 0 and 1 on $E_{01}$, then $I_{1}\left(E_{01}\right)$ is a strong relative maximum when $u_{3}$ is not allowed to vary, and $I_{2}\left(E_{01}\right)$ is a strong relative maximum when $u_{1}$ and $u_{2}$ are not allowed to vary.

The conditions for a weak relative maximum do not require that the Weierstrass conditions be satisfied.

THEOREM 9. If $E_{01}$ is an extremal arc and if the Legendre conditions (16) and (17) without the equality sign are satisfied at every set of values $\left(u_{1}, u_{1}^{\prime}\right.$, $\left.\cdots, u_{4}, u_{4}^{\prime}, x\right)$ on this arc, and if there is no point 2 conjugate to 0 between 0 and 1 on $E_{01}$, then $I_{1}\left(E_{01}\right)$ is a weak relative maximum when $u_{3}$ is not allowed to vary, and $I_{2}\left(E_{01}\right)$ is a weak relative maximum when $u_{1}$ and $u_{2}$ are not allowed to vary.

* If $w(x)$ is a continuous function admitting a continuous derivative in $x_{0} \leqq x \leqq x_{1}$, we shall say that it belongs to the class (I) in the interval $\left(x_{0} x_{1}\right)$. See E. Goursat, Cours d'Analyse Mathematique, vol. 3, p. 547 .

$\dagger$ These are the classical definitions given by Goursat, loc. cit., pp. 612-613. 
Although the above sufficient conditions apply strictly to the generalized Lagrange problem, by a slight modification they can be made to apply to the classical problem where only one integral $I_{1}$ is considered. Thus, allowing $u_{3}$ to vary in $F_{1}$ requires that the subscript $k$ in (14) and (15) take on the values 1, 2, 3 instead of 1 and 2 only. The arguments of $F_{1}$ and $G$ in all of the relations must of course be changed so that $U_{3}^{\prime}$ is given a proper place. In as much as this change will be obvious to the careful reader no attempt to write the corresponding conditions for the classical problem will be made here.

The sufficiency theorems for the general problem when one end point is variable differ from those just given in that the transversality conditions must be adjoined.*

\section{INTEGRAL EQUATION TREATMENT OF THE PROBLEM OF LAGRANGE FOR MORE THAN ONE DIFFERENTIAL EQUATION}

11. Equations of variation. It is readily seen that the analysis of the preceding chapter applies to the problem of Lagrange for one differential equation. By introducing the theory of Volterra integral equations $\dagger$ this analysis can be modified to apply to the Lagrange problem for more than one differential relation and to the more general problem for which the differential relation is replaced by a functional relation of the type referred to in the introduction. Since the method employed in solving the problem for two differential equations is perfectly general, we need only discuss this case.

Our problem is to determine, through two fixed points 0 and 1 in the hyperspace $\left(u_{1}, u_{2}, u_{3}, x\right)$, a curve $E_{01}$ which satisfies two differential equations $G_{k}\left(u_{1}, u_{1}^{\prime}, u_{2}, u_{2}^{\prime}, u_{3}, u_{3}^{\prime}, u_{4}, u_{4}^{\prime}, x\right)=0, k=1,2$, and which furnishes a maximum for an integral

$$
I=\int_{x_{0}}^{x_{1}} F\left(u_{1}, u_{1}^{\prime}, u_{2}, u_{2}^{\prime}, u_{3}, u_{3}^{\prime}, u_{4}, u_{4}^{\prime}, x\right) d x .
$$

We assume the $G_{k}$ to be functionally independent, i.e.

$$
\Delta G=\left|\begin{array}{ll}
\partial G_{1} / \partial u_{2}^{\prime} & \partial G_{1} / \partial u_{3}^{\prime} \\
\partial G_{2} / \partial u_{2}^{\prime} & \partial G_{2} / \partial u_{3}^{\prime}
\end{array}\right| \neq 0
$$

in the region under consideration, and to possess continuous second-order partial derivatives with respect to $u_{1}, u_{1}^{\prime}, u_{2}, u_{2}^{\prime}, u_{3}, u_{3}^{\prime}, u_{4}, u_{4}^{\prime}, x$. Although

* Bliss, loc. cit., pp. 169-170.

$\dagger$ For the theory of Volterra integral equations see V. Volterra, Legons sur les Equations Integrales. 
we have chosen both ends fixed, it is not necessary to make this assumption, as will appear presently. Let us first consider the problem stated above for both end points fixed.

Let the maximizing curve $E_{01}$, if such a curve exist, be the one defined by the equations

$$
\begin{array}{lr}
u_{p}=z_{p}(x) & (p=1,2), \\
u_{3}=y(x) & \left(x_{0} \leqq x \leqq x_{1}\right)
\end{array}
$$

and write

$$
u_{3}=y+\theta(x, a) ; u_{p}=z_{p}+f_{p}(x, a) \quad(p=1,2),
$$

where $\theta$ and $f_{p}$ are functions continuous with their second derivatives with respect to $x$ and $a$, and which vanish when $a$ vanishes. This notation for the $u_{i}, i=1,2,3$, is used to indicate that $u_{3}$ is to be regarded as the function whose variation is independent. We assumc the variations of $u_{1}$ and $u_{2}$ to be determined by the end values $u_{1}\left(x_{0}\right)=u_{10}, u_{2}\left(x_{0}\right)=u_{20}$ and the differential equations of total variation

$$
\begin{aligned}
\left(\partial G_{k} / \partial y\right) \delta \theta+\left(\partial G_{k} / \partial y^{\prime}\right) \delta \theta^{\prime}+\sum_{p=1}^{2}\left[\left(\partial G_{k} / \partial z_{p}\right) \delta f_{p}+\left(\partial G_{k} / \partial z_{p}^{\prime}\right) \delta f_{p}^{\prime}\right] & =0 \\
(k & =1,2)
\end{aligned}
$$

obtained by substituting the values of $u_{i}$ given above in the differential equations $G_{k}=0$, differentiating with respect to $a$ and then setting $a=0$. As we showed in $\S 3$ this is consistent with the assumption that 0 is a fixed end point.

If these same values of $u_{i}$ be substituted in $F$, the integral $I$ becomes a function of the parameter $a$ and yields on differentiation with respect to this parameter

$$
(\partial I / \partial a) \delta a=\int_{x_{0}}^{x_{1}}\left[(\partial F / \partial y) \delta \theta+\left(\partial F / \partial y^{\prime}\right) \delta \theta^{\prime}+\left(\partial F / \partial z_{r}\right) \delta f_{r}+\left(\partial F / \partial z_{r}^{\prime}\right) \delta f_{r}^{\prime}\right] d x,
$$

where $r$ is an umbral index with range 1,2 corresponding to $p$ with range 1, 2 .

12. Dependent variations by the theory of Volterra integral equations. In the classical treatment of this problem, Lagrange multipliers are introduced at this stage, but they can be advantageously avoided by integrating equation (19) with respect to $x$ between the limits $x_{0}$ and $x$, where $x_{0} \leqq x \leqq x_{1}$. If we perform this integration, replace $x$ under the integral sign by $s$, and then perform an integration by parts on the terms which involve the primed variations, we obtain 


$$
\begin{aligned}
\left(\partial G_{k} / \partial z_{r}^{\prime}\right) \delta f_{r}+\left(\partial G_{k} / \partial y^{\prime}\right) \delta \theta & +\int_{x_{0}}^{x}\left[\partial G_{k} / \partial y-\frac{d}{d s}\left(\partial G_{k} / \partial y^{\prime}\right)\right] \delta \theta d s \\
& +\int_{x_{0}}^{x}\left[\partial G_{k} / \partial z_{r}-\frac{d}{d s}\left(\partial G_{k} / \partial z_{r}^{\prime}\right)\right] \delta f_{r} d s=0,
\end{aligned}
$$

for, since $\delta \theta$ vanishes at $x_{0}$, the $\delta f_{r}$ must also vanish if the determinant $\Delta G$ is not zero in $x_{0} \leqq x \leqq x_{1}$. We take $r$ umbral as before.

The variations $\delta f_{r}$ are then determined by the system of Volterra integral equations

$$
\delta f_{r}(x)=\phi_{r}(x)+\int_{x_{0}}^{x} K_{r p}(x, s) \delta f_{p}(s) d s \quad(r=1,2),
$$

where by definition

$$
\begin{aligned}
\phi_{r}(x) & =A_{h r}(x)\left[\left(\partial G_{h} / \partial y^{\prime}\right) \delta \theta+\int_{x_{0}}^{x}\left[\partial G_{h} / \partial y-\frac{d}{d s}\left(\partial G_{h} / \partial y^{\prime}\right)\right] \delta \theta d s\right], \\
K_{r p}(x, s) & =A_{h r}(x)\left[\left(\partial G_{h}(s) / \partial z_{p}\right)-\frac{d}{d s}\left(\partial G_{h}(s) / \partial z_{p}^{\prime}\right)\right],
\end{aligned}
$$

where $h$ and $p$ are umbral indices having ranges 1,2 , and $A_{h r}$ is the cofactor of the corresponding element of $\Delta G$ divided by $-\Delta G$.

These integral equations form a Volterra system of the second type for the determination of the $\delta f_{r}$, uniquely, if the kernels $K_{r p}(x, s)$ are finite and integrable in the interval $x_{0} \leqq s \leqq x \leqq x_{1}{ }^{*}$

If $\Delta G \neq 0$ on the range $x_{0} \leqq s \leqq x \leqq x_{1}$, the $K_{r p}(x, s)$ will be finite and integrable on the range because of the continuity requirements on the $G_{k}$. The unique solution of the system is, therefore,

$$
\delta f_{r}(x)=\phi_{r}(x)+\int_{x_{0}}^{x} S_{r p}(x, s) \phi_{p}(s) d s,
$$

where $p$ is umbral with range 1,2 and $S_{r p}(x, s)$ is the resolvent kernel of $K_{r p}(x, s)$ defined by the equations

$$
\begin{aligned}
& K_{r p}^{1}(x, s)=-K_{r p}(x, s), \\
& K_{r p}^{i}(x, s)=\int_{0}^{x} K_{r h}^{\sigma}(x, t) K_{h p}^{i}(i, s) d t \\
& S_{r p}(x, s)=\sum_{i=1}^{\infty} K_{r p}^{i}(x, s) .
\end{aligned}
$$

* Volterra, loc. cit., p. 71. 
If we substitute in (22) the values of $\phi_{r}(x)$ and $\phi_{p}(s)$ as given by their definitions, then apply Dirichlet's formula to the iterated integral of the result, and then interchange the parameters of integration, we may write the variation of $\delta f_{r}$ as

$$
\delta f_{r}(x)=W_{r}(x) \delta \theta+\int_{x_{0}}^{x} V_{r}(x, s) \delta \theta d s,
$$

where by definition

$$
\begin{gathered}
W_{r}(x)=A_{h r}(x)\left(\partial G_{h} / \partial y^{\prime}\right), \\
V_{r}(x, s)=A_{h r}(x)\left[\partial G_{h} / \partial y-\frac{d}{d s}\left(\partial G_{h} / \partial y^{\prime}\right)\right]+A_{h p}(s) S_{r p}(x, s)\left(\partial G_{h} / \partial y^{\prime}\right) \\
+A_{h p}(s)\left[\partial G_{h} / \partial y-\frac{d}{d s}\left(\partial G_{h} / \partial y^{\prime}\right)\right] \int_{0}^{x} S_{r p}(x, t) d t
\end{gathered}
$$

and where $h$ and $p$ are umbral indices with range 1,2 .

By differentiation with respect to $x$ we obtain

$$
\delta f_{r}^{\prime}(x)=\frac{d}{d x}\left[W_{r} \delta \theta\right]+V_{r}(x, x) \delta \theta+\int_{x_{0}}^{x}\left[\partial V_{r}(x, s) / \partial x\right] \delta \theta d s .
$$

13. Eulerian equations. A substitution of $\delta f_{r}(x)$ and $\delta f_{r}^{\prime}(x)$ in the first variation of $I$ followed by an application of Dirichlet's formula as before yields

$$
\begin{aligned}
(\partial I / \partial a) \delta a= & \int_{x_{0}}^{x_{1}}\left[\partial F / \partial y+\left(\partial F / \partial z_{r}\right) W_{r}(x)+V_{r}(x, x)\left(\partial F / \partial z_{r}^{\prime}\right)+T(x)\right] \delta \theta d x \\
& +\int_{x_{0}}^{x_{1}}\left[\left(\partial F / \partial y^{\prime}\right) \delta \theta^{\prime}+\left(\partial F / \partial z_{r}^{\prime}\right) d\left(W_{r} \delta \theta\right) / d x\right] d x,
\end{aligned}
$$

where

$$
T(x)=\int_{x_{0}}^{x_{1}}\left[\left(\partial F / \partial z_{r}\right) V_{r}(s, x)+\left(\partial F / \partial z_{r}^{\prime}\right)\left(\partial V_{r}(s, x) / \partial s\right)\right] d s .
$$

An integration by parts performed on the primed terms yields

$$
\begin{aligned}
\delta I=\int_{x_{0}}^{x_{1}}\left[\partial F / \partial y+\left(\partial F / \partial z_{r}\right) W_{r}+V_{r}(x, x)\left(\partial F / \partial z_{r}^{\prime}\right)\right. & +T(x)-\frac{d}{d x}\left(\partial F / \partial y^{\prime}\right) \\
& \left.-W_{r} \frac{d}{d x}\left(\partial F / \partial z_{r}^{\prime}\right)\right] \delta \theta d x .
\end{aligned}
$$

In order that $\delta I$ vanish for all $\delta \theta$ it is necessary that the coefficient of $\delta \theta$ in the above integral vanish and hence 


$$
\begin{aligned}
\partial F / \partial y+\left(\partial F / \partial z_{r}\right) W_{r}+V_{r}(x, x)\left(\partial F / \partial z_{r}^{\prime}\right) & +T(x)-\frac{d}{d x}\left(\partial F / \partial y^{\prime}\right) \\
& -W_{r} \frac{d}{d x}\left(\partial F / \partial z_{r}^{\prime}\right)=0,
\end{aligned}
$$

where $r$ is an umbral index with range 1,2 .

It is well to note that the function $T(x)$ is an integral involving the resolvent kernel of the system of Volterra integral equations defining the variations. If some variable other than $u_{3}$ had been chosen to be independent, a different set of conditions of the type (25) would result, but presumably the new set would be equivalent to (25).

If one or both end points were variable, the problem could still be treated by the methods of this paragraph. For this case equation (20) would contain terms in $\delta \theta\left(x_{0}\right), \delta \theta\left(x_{1}\right), \delta f_{r}\left(x_{0}\right), \delta f_{r}\left(x_{1}\right)$. These terms could be carried all the way through the analysis and would yield transversality conditions in a new form. This interesting problem will not be attacked in the present paper.

\section{FURTher genERALIzations}

14. Problem for functional relations. A special problem in which a linear integral equation replaces the first-order differential equation $G=0$ has already been considered.* We desire now to consider the more general problem of determining a curve $E_{01}$ of the space $\left(u_{1}, u_{2}, u_{3}, x\right)$ satisfying functional relations

$$
\begin{aligned}
& G_{k}\left(u_{1}, u_{1}^{\prime}, u_{2}, u_{2}^{\prime} ; u_{3}, u_{3}^{\prime}, x\right) \\
& \quad=\int_{x_{0}}^{x} P_{k}\left(u_{1}, u_{1}^{\prime}, u_{2}, u_{2}^{\prime}, u_{3}, u_{3}^{\prime}, x, s\right) d s \quad(k=1,2),
\end{aligned}
$$

such that an integral

$$
I=\int_{x_{n}}^{x_{1}} F\left(u_{1}, u_{1}^{\prime} ; u_{2}, u_{2}^{\prime}, u_{3}, u_{3}^{\prime}, x\right) d x
$$

is a maximum. We may suppose the end parameter $x_{0}$ and the corresponding end values $u_{i}\left(x_{0}\right), i=1,2,3$, to be fixed, although this is not necessary. Let us, for the sake of brevity, also suppose $x_{1}$ and the corresponding end values of the $u_{i}$ to be fixed.

If the $u_{i}$ are replaced by functions satisfying the same conditions as the corresponding functions of $\$ 11$, the functional equations (26) become relations involving the parameter $a$ and yield by parametric differentiation

\footnotetext{
- A mathematical theory of competition, loc. cit., p. 173.
} 


$$
\begin{aligned}
\left(\partial G_{k} / \partial y\right) \delta \theta+\left(\partial G_{k} / \partial y^{\prime}\right) \delta \theta^{\prime}+\left(\partial G_{k} / \partial z_{r}\right) \delta f_{r}+\left(\partial G_{k} / \partial z_{r}^{\prime}\right) \delta f_{r}^{\prime} & \\
= & \int_{z_{r}}^{x}\left[\left(\partial P_{k} / \partial y\right) \delta \theta+\left(\partial P_{k} / \partial y^{\prime}\right) \delta \theta^{\prime}\right. \\
& \left.+\left(\partial P_{k} / \partial z_{r}\right) \delta f_{r}+\left(\partial P_{k} / \partial z_{r}^{\prime}\right) \delta f_{r}^{\prime}\right] d s \quad(k=1,2)
\end{aligned}
$$

where $r$ is umbral with range 1,2 .

An integration with respect to $x$ followed by an integration by parts on the primed variations yields

$$
\begin{aligned}
\left(\partial G_{k} / \partial y^{\prime}\right) \delta \theta+ & \int_{x_{0}}^{x}\left[\partial G_{k} / \partial y-\frac{d}{d s}\left(\partial G_{k} / \partial y^{\prime}\right)\right] \delta \theta d s+\left(\partial G_{k} / \partial z_{r}^{\prime}\right) \delta f_{r} \\
+ & \int_{x_{0}}^{x}\left[\partial G_{k} / \partial z_{r}-\frac{d}{d s}\left(\partial G_{k} / \partial z_{r}^{\prime}\right)\right] \delta f_{r} d s \\
= & \int_{x_{0}}^{x}\left[\left(\partial P_{k} / \partial y^{\prime}\right) \delta \theta+\left(\partial P_{k} / \partial z_{r}^{\prime}\right) \delta f_{r}\right] d s \\
& +\int_{x_{0}}^{x} d s \int_{x_{0}}^{\circ}\left[\left(\partial P_{k} / \partial y-\frac{d}{d t}\left(\partial P_{k} / \partial y^{\prime}\right)\right) \delta \theta\right. \\
& \left.+\left(\partial P_{k} / \partial z_{r}-\frac{d}{d t}\left(\partial P_{k} / \partial z_{r}^{\prime}\right)\right) \delta f_{r}\right] d t
\end{aligned}
$$

where for convenience in notation the parameter of integration $x$ has been changed to $s$.

If we apply Dirichlet's tormula to the iterated integral, we can write this expression as

$$
\begin{gathered}
\left(\partial G_{k} / \partial z_{r}^{\prime}\right) \delta f_{r}=-\left(\partial G_{k} / \partial y^{\prime}\right) \delta \theta-\int_{x_{0}}^{x}\left[\partial G_{k} / \partial y-\frac{d}{d s}\left(\partial G_{k} / \partial y^{\prime}\right)-\partial P_{k} / \partial y^{\prime}\right. \\
\left.-(x-s)\left(\partial P_{k} / \partial y-\frac{d}{d s}\left(\partial P_{k} / \partial y^{\prime}\right)\right)\right] \delta \theta d s \\
-\int_{x_{0}}^{x}\left[\partial G_{k} / \partial z_{r}-\frac{d}{d s}\left(\partial G_{k} / \partial z_{r}^{\prime}\right)-\partial P_{k} / \partial z_{r}^{\prime}\right. \\
\left.-(x-s)\left(\partial P_{k} / \partial z_{r}-\frac{d}{d s}\left(\partial P_{k} / \partial z_{r}^{\prime}\right)\right)\right] \delta f_{r} d s .
\end{gathered}
$$

As far as the variations of $\delta f_{r}$ and $\delta \theta$ are concerned this expression is of the same form as (21), if $\partial G_{k} / \partial z_{r}^{\prime}$ is not zero on the range $x_{0} \leqq s \leqq x \leqq x_{1}$. The analysis of the preceding section, therefore, applies from this point. 
15. Further extensions. The extension to the case of more than one independent variable is obtained by placing a subscript on $y$ in the above equations and regarding this subscript as an umbral index of the proper range.

The problem of simultaneous maxima for more than one differential or integral relation can be treated by this same method, since, if there are two integrals and two independent variables $u_{3}$ and $u_{4}$, equation (25), with the proper arguments for $F_{1}, F_{2}$ and $G_{k}$ substituted, is a necessary condition that a curve $E_{01}$ in the space $\left(u_{1}, u_{2}, u_{3}, u_{4}, x\right)$ satisfy differential equations $G_{k}\left(u_{1}, u_{1}^{\prime}, \cdots, u_{4}, u_{4}^{\prime}, x\right)=0, k=1,2$, and make an integral

$$
I=\int_{x}^{x_{1}} F_{1}\left(u_{1}, u_{1}^{\prime}, \cdots, u_{4}, u_{4}^{\prime}, x\right) d x
$$

a maximum when $u_{4}$ is not allowed to vary.

A discussion of the corresponding problems for variable end points should lead to analogues of the Weierstrass and Legendre necessary conditions and to sufficient conditions for strong and weak relative maxima.

The assumption that the admissible arcs have continuously turning tangents is by no means necessary. If we make assumptions on admissible arcs similar to those of Part II, we can obtain the analogues of the more general Euler equations (7) and (8), by integrating by parts the terms in $\delta \theta$ instead of those in $\delta \theta^{\prime}$. The analogues of the Weierstrass-Erdmann corner conditions* follow readily from the Euler equations in the form (7) and (8).

* Bliss, loc. cit., p. 143, and O. Bolza, Vorlesungen über Variationsrechmung, 1909, p. 366.

RICE INSTITUTE,

Houston, Texas 